\title{
Clinical Evaluation of TRICOM Vector Therapeutic Cancer Vaccines
}

Ravi A. Madan, M.D.,

Assistant Clinical Investigator, Medical Oncology Branch, Center for Cancer Research, National Cancer Institute, National Institutes of Health, Bethesda, MD

Marijo Bilusic, M.D., Clinical Fellow, Medical Oncology Branch, Center for Cancer Research, National Cancer Institute, National Institutes of Health, Bethesda, MD

Christopher Heery, M.D.,

Clinical Fellow, Medical Oncology Branch, Center for Cancer Research, National Cancer Institute, National Institutes of Health, Bethesda, MD

Jeffrey Schlom, Ph.D., and

Laboratory Chief, Laboratory of Tumor Immunology and Biology, Center for Cancer Research, National Cancer Institute, National Institutes of Health, Bethesda, MD

James L. Gulley, M.D., Ph.D. Laboratory of Tumor Immunology and Biology and Medical Oncology Branch, Center for Cancer Research, National Cancer Institute, National Institutes of Health, Bethesda, MD

\section{Abstract}

We have developed an "off-the-shelf" vector-based vaccine platform containing transgenes for carcinoma-associated antigens and multiple costimulatory molecules (designated TRICOM). Two TRICOM platforms have been evaluated both preclinically and in clinical trials. PROSTVAC consists of $\mathrm{rV}$, rF-PSA-TRICOM and is being used in prostate cancer therapy trials. PANVAC consists of $\mathrm{rV}, \mathrm{rF}-\mathrm{CEA}-\mathrm{MUC1}$-TRICOM; the expression of the two pan-carcinoma transgenes CEA and MUC-1 renders PANVAC vaccination applicable for therapeutic applications for a range of human carcinomas. Many new paradigms have emerged as a consequence of completed and ongoing TRICOM vaccine trials, including (a) clinical evidence of patient benefit may be delayed, because multiple vaccinations may be necessary to induce a sufficient anti-tumor immune response; (b) survival, and not strict adherence to RECIST criteria or time-to-progression, may be the most appropriate trial endpoint when TRICOM vaccines are used as monotherapy; (c) certain patient populations are more likely to benefit from vaccine therapy as compared to other therapeutics; and (d) TRICOM vaccines combined with standard-of-care therapeutics, either concomitantly or sequentially, are feasible because of the limited toxicity of vaccines.

Address correspondence to Jeffrey Schlom, Ph.D., Laboratory of Tumor Immunology and Biology, Center for Cancer Research, National Cancer Institute, National Institutes of Health, 10 Center Drive, Room 8B09, MSC 1750, Bethesda, MD 20892. schlomj@mail.nih.gov.

Publisher's Disclaimer: This is a PDF file of an unedited manuscript that has been accepted for publication. As a service to our customers we are providing this early version of the manuscript. The manuscript will undergo copyediting, typesetting, and review of the resulting proof before it is published in its final citable form. Please note that during the production process errors may be discovered which could affect the content, and all legal disclaimers that apply to the journal pertain. 


\section{Strategic Plan}

Our program has focused on the design and development of vaccines for the therapy of human carcinomas. While some carcinoma types express distinct tumor-associated antigens (TAAs), such as PSA for prostate cancer, there are numerous TAAs, such as MUC-1 and CEA, that are shared among different carcinoma types. ${ }^{1-2}$ We have focused on the development of "off-the-shelf" vaccines that can be widely evaluated at numerous institutions. As will be discussed below, clinical trial data are now emerging that the optimal use of cancer vaccines as monotherapy will be early in the disease process and in patients with low tumor burden metastatic disease. ${ }^{3-4}$ Clinical trials with several types of vaccines and other immunotherapeutics are now demonstrating that as monotherapy the appropriate endpoint should be survival and not restricted to RECIST criteria. ${ }^{5}$ Perhaps most importantly we look upon the future of cancer vaccine therapy as part of an immuneoncology platform combining vaccines with other immune-based therapies and with certain chemotherapeutic agents, local radiation of tumor, and some small molecule targeted therapies. An important property of cancer vaccines that is often overlooked is their negligible toxicity profile. This attribute allows their use in the adjuvant and neo-adjuvant setting, and in combination with other therapeutic modalities where toxicities of both agents are not compounding.

\section{TRICOM Vaccine Platform}

We have developed a vaccine platform ${ }^{6-13}$ consisting of a recombinant vaccinia virus ( $\left.\mathrm{rV}^{-}\right)$ prime and multiple recombinant avipox (fowlpox, $\mathrm{rF}-$ ) booster vaccinations (Figure 1). Each vector contains transgenes for one or more TAAs and transgenes for three costimulatory molecules (B7.1 (CD80), ICAM-1 (CD54), and LFA-3 (CD58), designated TRICOM) to enhance immune responses. Vaccinia is the agent used in the smallpox vaccine, whih has been administered to over 1 billion people in the worldwide eradication of smallpox. ${ }^{14}$ The use of a recombinant vaccinia induces a strong immune response to the TAA transgene but can be used only once due to host-neutralizing immunity to the virus. ${ }^{15}$ Avipox, on the other hand, is an avian virus that is replication defective in mammals; it has been shown in both preclinical $^{15}$ and clinical studies ${ }^{16-17}$ to be used multiple times with little if any hostneutralizing immunity. Both vectors do not integrate into host DNA and efficiently infect antigen-presenting cells (APCs) such as dendritic cells. Extensive preclinical studies have shown ${ }^{6-7}, 18,8$ that the three costimulatory molecule transgenes act synergistically to greatly enhance immune responses to tumor antigens, and to enhance consequent anti-tumor efficacy with no adverse toxicity in numerous preclinical models. Two TRICOM platforms have been developed and evaluated in clinical trials. PROSTVAC consists of $\mathrm{rV}$, rF-PSATRICOM and is being used in prostate cancer therapy trials. ${ }^{19,3,20}$ PANVAC consists of $\mathrm{rV}$, rF-CEA-MUC1-TRICOM; ${ }^{21-23}$ the expression of the two pan-carcinoma transgenes CEA and MUC-1 renders PANVAC vaccination applicable for therapeutic applications for a range of human carcinomas including colorectal, breast and ovarian carcinomas.

\section{Prostate Cancer as a Target for Vaccine Therapy}

Several characteristics make prostate cancer an acceptable target for vaccine-mediated therapy. Time may be required to generate an immune response capable of curtailing disease growth. Prostate cancer is generally an indolent disease that may not lead to death for over a decade after diagnosis and even several years after metastasis. Prostate tumor cells also overexpress several antigens which can be identified by immune cells; these include PSA, prostatic acid phosphatase (PAP), TARP and NGEP. ${ }^{24}$ In addition, PSA as a serum marker may be valuable as it enables clinicians to identify patients with minimal tumor burden and thus at a time when they may be ideal candidates for vaccine therapy. 
Two therapeutic cancer vaccines have demonstrated an overall survival advantage in metastatic castrate-resistant prostate cancer (mCRPC). Sipuleucel- $\mathrm{T}^{25}$ represents an alternative strategy to the TRICOM poxviral vaccines and is a patient-specific APC-based vaccine generated from the peripheral blood of each individual patient. Once collected via leukapheresis, the peripheral blood cells are transported to a central processing facility where they are pulsed in vitro by a PAP/GM-CSF fusion protein. Cells are then transported back for vaccine infusion. Although a pair of small phase III trials failed to meet their primary endpoint of improved time to disease progression, there was evidence that Sipuleucel-T prolonged survival in mCRPC. ${ }^{26}$ A larger phase III trial was then conducted with overall survival as the end point, enrolling more than 500 patients and randomizing them 2:1 in favor of Sipuleucel-T. Again, no change in time to progression was seen, but overall survival was improved in the vaccine arm ( 25.8 months vs. 21.7 months; $P=$ 0.032). ${ }^{25}$ In April 2010, the FDA approved Sipuleucel-T for the treatment of minimal or non-symptomatic mCRPC.

We have designed and developed the "off-the-shelf" PSA-TRICOM vaccine platform ${ }^{3,20}$ (Figure 1). A phase I trial with monthly administered PSA-TRICOM demonstrated safety, with local site reactions being the most common adverse event. ${ }^{19}$ A randomized 43 -center phase II trial enrolled 125 minimally symptomatic mCRPC patients, randomizing them 2:1 in favor of vaccine. Similar to the experience with Sipuleucel-T, PSA-TRICOM did not alter time to progression (the primary endpoint), yet improved median overall survival relative to control vector placebo, 25.1 and 16.6 months ( $\mathrm{p}=0.0061$; Figure 2 ) ${ }^{20} \mathrm{~A}$ smaller phase II trial in $\mathrm{mCRPC}$ at the NCI evaluated clinical and immunologic responses. ${ }^{3}$ The median overall survival in this single arm study was 26.6 months, similar to the larger PSATRICOM trial. Patients with the greatest PSA-specific T-cell response post 3 vaccinations also had a substantial improvement in survival relative to those who had lesser PSA-specific T-cell responses $(\mathrm{p}=0.055){ }^{3}$ A retrospective analysis evaluated patients based on the Halabi nomogram, which was developed from over 1,100 mCRPC patients treated on Cancer and Leukemia Group B and designed to predict survival on chemotherapy or second-line hormonal therapy. ${ }^{27}$ The findings suggested that patients with more indolent disease characteristics and a predicted survival greater than 18 months using the Halabi nomogram ${ }^{27}$ had greater improvements in survival (compared to that predicted employing chemotherapy or second-line hormonal therapy) than patients with more aggressive disease characteristics (Table 1). ${ }^{3}$

This is contrasted with a contemporary trial at NCI with a similar patient population in which the Halabi nomogram ${ }^{27}$ more accurately predicted response to chemotherapy (docetaxel) (Table 1). These data provide insight into the planning of future trials employing PSA-TRICOM vaccine monotherapy. An international phase III trial of PSA-TRICOM in mCRPC began enrolling patients in late 2011.

\section{Kinetics of Clinical Response after Therapeutic Vaccination}

With traditional cytotoxic agents, it is widely believed that improved time to progression is a prerequisite for improved overall survival; this is certainly intuitive. Cytotoxic agents affect the tumor only during the period of administration; soon after the drug is discontinued, its antitumor activity ends. By contrast, with active immunotherapies, the mechanism of action and kinetics of clinical response appear to be quite different. Therapeutic vaccines do not directly target the tumor, but rather target the immune system, which in turn targets the tumor. That immune response can be enhanced by continued booster vaccinations, increasing immune-mediated tumor cell lysis killing. This in turn can lead to cross-priming of TAAs, thus broadening the immune repertoire (a phenomenon known as antigen cascade or epitope spreading). This broader, and perhaps more relevant, immune response may take 
some time to develop. Thus, an antitumor immune response can induce memory cells that may provide ongoing antitumor activity long after the vaccine is given. Although they may not bring about a significant reduction in tumor burden, therapeutic vaccines can apply continuous antitumor activity over a long period of time, resulting in a slower tumor growth rate. This deceleration in growth rate may take weeks or months to commence, but may continue for months or years and, more importantly, through subsequent therapies. This process can lead to clinically significant improved overall survival, often with no difference in time to progression and a low rate of objective responses; detailed descriptions of these concepts have recently been published. ${ }^{28-29}$ The concept of slowing the rate of tumor growth suggests that treating patients with immunotherapy earlier rather than later in the course of disease may result in far better outcomes. Thus, patients treated with vaccines within the last 6 to 12 months of life (a typical phase I patient population) may not experience any clinically relevant improvement in survival from a slightly altered growth rate, whereas those with a lower tumor burden treated earlier in their disease course may eventually experience a significant improvement in overall survival, even with the same modest decrease in the rate of tumor growth. ${ }^{4}$

\section{Reduced Toxicity is Important}

It is also important to note that the TRICOM and Sipuleucel-T vaccines carry significantly less toxicity than other agents that improve survival in mCRPC (Table 2). Other therapeutics demonstrating a survival advantage in mCRPC including docetaxel, cabazitaxel, and abiraterone demonstrate a rate of discontinuation due to adverse events (between $11 \%$ and $19 \%)$. This is contrasted with approximately $2 \%$ of patients who discontinue vaccine due to toxicity in trials of PSA-TRICOM and Sipuleucel-T (Table 2). ${ }^{20,25,}$, 64, 65, 66

\section{New Paradigms Emerging for Therapeutic Cancer Vaccines}

When used as monotherapy, it is now becoming apparent from vaccine therapy clinical studies that defining the appropriate patient population is of great importance. Early phase III trials demonstrated that the patients least likely to generate immune responses to vaccine were those who had received the most rounds of prior chemotherapy and the shortest interval since their last chemotherapeutic regimen. ${ }^{30-31}$ This is not to be confused with the use of other therapeutics concurrent with vaccine, or following vaccine, as will be discussed below.

A classic paradigm paralysis continues with the insistence by some that only after tumor lesions are reduced using classic RECIST criteria can a therapeutic modality be considered beneficial. Indeed, clinical studies with small molecule targeted therapeutics, as well as with different immunotherapeutic agents, have demonstrated increased survival in randomized studies with minimal, if any, reduction in tumor burden or time to progression. ${ }^{32,2} \mathrm{In}$ addition to the PSA-TRICOM and Sipuleucel-T trials described above, a phase III trial with MAb anti-CTLA4 in patients with metastatic melanoma ${ }^{33}$ demonstrated a statistically significant advantage in survival with lack of a statistical difference in time to progression. There are at least two possible yet inclusive explanations for this phenomenon. An examination $^{29}$ of five clinical trials (four with chemotherapy and one with PSA-TRICOM vaccine) conducted at $\mathrm{NCI}$ in patients with metastatic prostate cancer demonstrated that with the use of chemotherapy there was an initial tumor reduction, but that the growth rate of tumors at relapse was similar to the initial tumor growth rate prior to any therapy (Figure $3 \mathrm{~A}$, line (b)); this is contrasted with the reduction in tumor growth rate following vaccine therapy (Figure 3A, line (c)). ${ }^{29}$ Thus for patients with little or any tumor reduction (and thus virtually no increase in time to progression), an increased survival was observed. This phenomenon could potentially be enhanced if vaccine therapy is initiated earlier in disease 
progression or in patients with low tumor burden metastatic disease (Figure 3B, line (d)), while little or no effect is seen if vaccine therapy is initiated in patients with large tumor burden (Figure 3B, line (e)). Additional therapies received with vaccine may take advantage of both modalities (Figure 3C). This will be discussed below.

Unlike other forms of cancer therapy, which are passive and whose effect is usually immediate, it often takes numerous vaccinations to bolster the patient's immune response (Table 3). It has been demonstrated in both vaccine trials and trials with anti-CTLA4 that anti-tumor responses can be delayed post-therapy. ${ }^{33,25,20}$ Early clinical trials with vaccine may thus well have been terminated prematurely with the observance of tumor progression before sufficient vaccine boosts could be administered. This phenomenon has actually led to modifications in how vaccine clinical trials are now designed and to "new immune response criteria" for immunotherapy. ${ }^{34}$

\section{Separating Vaccine Efficacy from Poor Clinical Trial Design}

A classic example of the distinction between a vaccine's potential efficacy and a poor clinical trial design was evidenced by an ill-conceived corporate phase III trial in which PANVAC vaccine (Therion Biologics, Cambridge, MA, U.S.A.) was administered to patients with metastatic pancreatic cancer who had already failed prior gemcitabine therapy. ${ }^{22}$ As many predicted, this trial failed to meet its primary endpoint of overall survival. Poor clinical trial design was clearly illustrated by (a) the median overall survival of less than 3 months in this patient population; (b) numerous randomized trials of various FDA-approved chemotherapy combinations have failed to extend survival in this patient population of second-line pancreatic cancer; and (c) the best phase II data only demonstrate a median second-line survival of 4.8 months for treatment with oxaliplatin, folinic acid and 5-FU vs. 2.3 months with best supportive care. ${ }^{35}$ Thus, failure of a phase III vaccine trial in second-line therapy of pancreatic cancer should foremost be considered a failure in clinical trial design employing an inappropriate patient population for vaccine monotherapy.

\section{Tumor Size Does Matter}

Numerous preclinical studies have demonstrated that once a tumor reaches a certain volume, vaccine monotherapy will have limited effectiveness. Clinical data by Morse and Lyerly ${ }^{36-37}$ are now emerging that vaccine monotherapy will be most beneficial in patients with limited disease volume. A recent clinical trial employing a poxviral vaccine platform targeting CEA $(\mathrm{rV}-, \mathrm{rF}-\mathrm{CEA}-\mathrm{TRICOM})$ in colorectal cancer (CRC) patients with large volume liver metastases showed no evidence of patient benefit. ${ }^{67}$ This is contrasted with the recent results emerging ${ }^{36-37}$ employing the PANVAC vaccine ( $\mathrm{rV}-$, rF-CEA-MUC1-TRICOM) in CRC patients following metastasectomy for liver or lung metastases. Patients with completely resected CRC metastases remain at high risk of recurrence and death despite adjuvant chemotherapy. ${ }^{38-43}$

In a multicenter trial, ${ }^{36-37}$ patients $(n=74)$ with no evidence of disease after resection of CRC metastases and completion of their physician-determined perioperative chemotherapy were randomized 1:1 to four vaccinations with PANVAC, or PANVAC-modified dendritic cells (DC). Data from a prospectively registered, comparable, contemporary control group of CRC patients who had undergone metastasectomy were also available. The two vaccine arms and the contemporary controls were well balanced. The 2-year relapse-free survival (RFS) was similar in all groups: $50 \%$ for the DC-PANVAC group, $56 \%$ for the PANVAC group, and $55 \%$ for the contemporary control group. However, at a follow-up at approximately 40 months, ${ }^{36-37}$ there were $2 / 37$ deaths in the DC-PANVAC group and 5/37 in the PANVAC alone group, for a total of approximately $10 \%$ deaths (7/74, i.e., a $90 \%$ overall survival at 40 months); this is in contrast to approximately $58 \%$ deaths in the 
contemporary control group and with 3-year survival data of CRC patients postmetastasectomy from five other trials. ${ }^{38-39,} 41-43$ It is emphasized that this must be considered preliminary data in that the vaccine arms were not randomized to the contemporary control arm; thus a randomized Phase III study is warranted to confirm these results. It is of interest, however, that this is still another example of a vaccine trial showing little or no evidence of RFS, but with an apparent benefit in overall survival.

\section{TRICOM Vaccine Combination Therapies}

The limited toxicity induced by cancer vaccines makes them ideal for combination therapies. The use of vaccines with other immune-mediating therapies can be divided into several categories: vaccines with (a) enhancers of immune stimulation. This includes cytokines; (b) immune checkpoint inhibitors; (c) adoptive T-cell transfer therapy; and (d) other vaccines. For example, we have recently demonstrated ${ }^{44}$ that when two very distinct vaccine platforms (recombinant poxviral-TRICOM and recombinant yeast) are used in combination, distinct T-cell responses are observed using each vaccine platform, leading to enhanced antitumor effects. When and if randomized multi-center trials demonstrate the efficacy and feasibility of T-cell adoptive transfer therapies, there is a rationale for this strategy to be combined with vaccine therapy as we have demonstrated preclinically. ${ }^{45}$

Evidence is now emerging from preclinical studies for the use of therapeutic cancer vaccines with more conventional cancer therapies. ${ }^{46-48,12,49}$ Recent clinical data lends credence to the use of vaccines in combination with standard-of-care, FDA-approved therapeutics. A recently completed phase I study at the NCI in mCRPC demonstrated that when Ipilimumab (anti-CTLA4 MAb) was combined with PSA-TRICOM at escalating doses, the median survival was 34 months, which compares favorably to previous vaccine trials in mCRPC that resulted in median survivals of approximately 26 months. ${ }^{50}$ These and other data support the rationale that immune check point inhibitors may enhance the efficacy of therapeutic vaccines.

A series of hypothesis-generating randomized phase II trials at NCI are comparing standardof-care hormonal therapy, radiation therapy and chemotherapy alone, and in tandem with a poxviral TRICOM-based vaccine. In patients with non-metastatic CRPC, interval data favors patients receiving flutamide (an androgen receptor antagonist used as a second line hormonal therapy) with PSA-TRICOM, compared to flutamide alone. With half of the patients enrolled on this 62-patient trial, median time to progression with flutamide alone is 85 days vs. 233 days employing flutamide with PSA-TRICOM. ${ }^{51}$ In patients with advanced mCRPC who have already progressed on doctaxel, patients are randomized to single agent or Quadramet ${ }^{52}$ (chelated Samarium-153, a radiopharmaceutical that delivers localized radiation to bone metastasis and is FDA approved for palliation) vs. Quadramet plus PSATRICOM. In this trial now enrolling at 3 centers, there is a clear trend favoring Samarium-153 with PSA-TRICOM vs. Samarium-153 alone. ${ }^{53}$ In an ongoing trial, patients with metastatic breast cancer are randomized to either standard chemotherapy (docetaxel) alone vs. docetaxel plus PANVAC ( $\mathrm{rV}$, rF-CEA-MUC1-TRICOM), with time to progression as the endpoint. ${ }^{54}$ The hypothesis is that the combination therapy will take advantage of both modalities in tumor control (Figure 3C). At this time there is again an interval trend in time to progression favoring the vaccine combination arm.

There are many anecdotal reports and several publications implying that patients who have received vaccine therapy and then progressed undergo unexpected clinical responses with the administration of subsequent therapies. ${ }^{55-58}$ This phenomenon, however, has not been validated prospectively to date. A randomized ECOG multi-center clinical trial has recently been initiated in patients with metastatic prostate cancer to prospectively evaluate this 
phenomenon. ${ }^{59}$ Patients will receive either (a) docetaxel or (b) 2 months of PROSTVAC vaccine followed by docetaxel; survival will be the primary endpoint.

\section{Future Directions}

The TRICOM vaccine platforms (PROSTVAC and PANVAC) have demonstrated minimal toxicity in a wide range of carcinoma types, different stages of disease, and in combination with radiation, chemotherapy and hormone therapy. Future directions of TRICOM vaccines will involve their evaluation in the adjuvant and neo-adjuvant settings, as well as in patients with low burden metastatic disease. Being contemplated are trials in patients with a high risk of developing cancer or in patients with pre-neoplastic conditions, such as high grade prostatic intraepithelial neoplasia (PIN) and familial adenomatous polyposis (FAP). ${ }^{60-62}$

The "off-the-shelf" nature of this vaccine platform renders it exception suitable for multicenter randomized trials. While evidence of patient benefit has been seen in randomized phase II studies, only phase III trials in the appropriate patient populations and with the appropriate clinical endpoints for therapeutic vaccines will define if this vaccine platform has a place in cancer management either as a monotherapy or in combination therapies.

\section{Acknowledgments}

Funding Disclosures: The authors are supported by funds from the Intramural Research Program of the Center for Cancer Research, National Cancer Institute (NCI), NIH. In addition, there is a Collaborative Research and Development Agreement between the NCI/Laboratory of Tumor Immunology (LTIB) and Biology and Bavarian Nordic. Dr. Schlom is an inventor on several patents and patent applications related to therapeutic cancer vaccines.

The authors thank Debra Weingarten for her editorial assistance in the preparation of this manuscript.

\section{References}

1. Cheever MA, Allison JP, Ferris AS, Finn OJ, Hastings BM, Hecht TT, et al. The prioritization of cancer antigens: a national cancer institute pilot project for the acceleration of translational research. Clin Cancer Res. 2009; 15:5323-37. [PubMed: 19723653]

2. Gulley, JL.; Arlen, PM.; Hodge, JW.; Schlom, J. Chapter 57: Vaccines and immunostimulants. In: Kufe, D., editor. Holland-Frei Cancer Medicine. 8th. Shelton, CT: People's Medical Publishing House-USA; 2010. p. 725-36.p. 6

3. Gulley JL, Arlen PM, Madan RA, Tsang KY, Pazdur MP, Skarupa L, et al. Immunologic and prognostic factors associated with overall survival employing a poxviral-based PSA vaccine in metastatic castrate-resistant prostate cancer. Cancer Immunol Immunother. 2010; 59:663-74. [PubMed: 19890632]

4. Gulley JL, Madan RA, Schlom J. The impact of tumor volume on potential efficacy of therapeutic vaccines [review]. Curr Oncol. 2011; 18:e150-7. [PubMed: 21655153]

5. Therasse P, Eisenhauer EA, Verweij J. RECIST revisited: a review of validation studies on tumour assessment. Eur J Cancer. 2006; 42:1031-9. [PubMed: 16616487]

6. Hodge JW, Sabzevari H, Yafal AG, Gritz L, Lorenz MG, Schlom J. A triad of costimulatory molecules synergize to amplify T-cell activation. Cancer Res. 1999; 59:5800-7. [PubMed: 10582702]

7. Aarts WM, Schlom J, Hodge JW. Vector-based vaccine/cytokine combination therapy to enhance induction of immune responses to a self-antigen and antitumor activity. Cancer Res. 2002; 62:57707. [PubMed: 12384537]

8. Hodge JW, Grosenbach DW, Aarts WM, Poole DJ, Schlom J. Vaccine therapy of established tumors in the absence of autoimmunity. Clin Cancer Res. 2003; 9:1837-49. [PubMed: 12738742]

9. Yang S, Hodge JW, Grosenbach DW, Schlom J. Vaccines with enhanced costimulation maintain high avidity memory CTL. J Immunol. 2005; 175:3715-23. [PubMed: 16148117] 
10. Yang S, Tsang KY, Schlom J. Induction of higher-avidity human CTLs by vector-mediated enhanced costimulation of antigen-presenting cells. Clin Cancer Res. 2005; 11:5603-15. [PubMed: 16061879]

11. Chakraborty M, Schlom J, Hodge JW. The combined activation of positive costimulatory signals with modulation of a negative costimulatory signal for the enhancement of vaccine-mediated Tcell responses. Cancer Immunol Immunother. 2007; 56:1471-84. [PubMed: 17318654]

12. Garnett CT, Schlom J, Hodge JW. Combination of docetaxel and recombinant vaccine enhances Tcell responses and antitumor activity: effects of docetaxel on immune enhancement. Clin Cancer Res. 2008; 14:3536-44. [PubMed: 18519787]

13. Yang S, Schlom J. Antigen-presenting cells containing multiple costimulatory molecules promote activation and expansion of human antigen-specific memory CD8+ T cells. Cancer Immunol Immunother. 2009; 58:503-15. [PubMed: 18690438]

14. Fenner, F.; Henderson, D.; Arita, I. Smallpox and its eradication. Geneva: World Health Organization; 1988.

15. Hodge JW, Poole DJ, Aarts WM, Gomez Yafal A, Gritz L, Schlom J. Modified vaccinia virus ankara recombinants are as potent as vaccinia recombinants in diversified prime and boost vaccine regimens to elicit therapeutic antitumor responses. Cancer Res. 2003; 63:7942-9. [PubMed: 14633725]

16. Marshall JL, Hoyer RJ, Toomey MA, Faraguna K, Chang P, Richmond E, et al. Phase I study in advanced cancer patients of a diversified prime-and-boost vaccination protocol using recombinant vaccinia virus and recombinant nonreplicating avipox virus to elicit anti-carcinoembryonic antigen immune responses. J Clin Oncol. 2000; 18:3964-73. [PubMed: 11099326]

17. Marshall JL, Gulley JL, Arlen PM, Beetham PK, Tsang KY, Slack R, et al. Phase I study of sequential vaccinations with fowlpox-CEA(6D)-TRICOM alone and sequentially with vacciniaCEA(6D)-TRICOM, with and without granulocyte-macrophage colony-stimulating factor, in patients with carcinoembryonic antigen-expressing carcinomas. J Clin Oncol. 2005; 23:720-31. [PubMed: 15613691]

18. Greiner JW, Zeytin H, Anver MR, Schlom J. Vaccine-based therapy directed against carcinoembryonic antigen demonstrates antitumor activity on spontaneous intestinal tumors in the absence of autoimmunity. Cancer Res. 2002; 62:6944-51. [PubMed: 12460911]

19. Arlen PM, Skarupa L, Pazdur M, Seetharam M, Tsang KY, Grosenbach DW, et al. Clinical safety of a viral vector based prostate cancer vaccine strategy. J Urol. 2007; 178:1515-20. [PubMed: 17707059]

20. Kantoff PW, Schuetz TJ, Blumenstein BA, Glode LM, Bilhartz DL, Wyand M, et al. Overall survival analysis of a phase II randomized controlled trial of a Poxviral-based PSA-targeted immunotherapy in metastatic castration-resistant prostate cancer. J Clin Oncol. 2010; 28:1099_ 105. [PubMed: 20100959]

21. Tsang KY, Palena C, Yokokawa J, Arlen PM, Gulley JL, Mazzara GP, et al. Analyses of recombinant vaccinia and fowlpox vaccine vectors expressing transgenes for two human tumor antigens and three human costimulatory molecules. Clin Cancer Res. 2005; 11:1597-607. [PubMed: 15746065]

22. Madan RA, Arlen PM, Gulley JL. PANVAC-VF: poxviral-based vaccine therapy targeting CEA and MUC1 in carcinoma. Expert Opin Biol Ther. 2007; 7:543-54. [PubMed: 17373905]

23. Gulley JL, Arlen PM, Tsang KY, Yokokawa J, Palena C, Poole DJ, et al. Pilot study of vaccination with recombinant CEA-MUC-1-TRICOM poxviral-based vaccines in patients with metastatic carcinoma. Clin Cancer Res. 2008; 14:3060-9. [PubMed: 18483372]

24. Madan RA, Mohebtash M, Schlom J, Gulley JL. Therapeutic vaccines in metastatic castrationresistant prostate cancer: principles in clinical trial design. Expert Opin Biol Ther. 2010; 10:19-28. [PubMed: 19857185]

25. Kantoff PW, Higano CS, Shore ND, Berger ER, Small EJ, Penson DF, et al. Sipuleucel-T immunotherapy for castration-resistant prostate cancer. New Eng J Med. 2010; 363:411-22. [PubMed: 20818862]

26. Higano CS, Schellhammer PF, Small EJ, Burch PA, Nemunaitis J, Yuh L, et al. Integrated data from 2 randomized, double-blind, placebo-controlled, phase 3 trials of active cellular 
immunotherapy with sipuleucel-T in advanced prostate cancer. Cancer. 2009; 115:3670-9. [PubMed: 19536890]

27. Halabi S, Small EJ, Kantoff PW, Kattan MW, Kaplan EB, Dawson NA, et al. Prognostic model for predicting survival in men with hormone-refractory metastatic prostate cancer. J Clin Oncol. 2003; 21:1232-7. [PubMed: 12663709]

28. Madan RA, Gulley JL, Fojo T, Dahut WL. Therapeutic cancer vaccines in prostate cancer: the paradox of improved survival without changes in time to progression. Oncologist. 2010; 15:96975. [PubMed: 20798195]

29. Stein WD, Gulley JL, Schlom J, Madan RA, Dahut W, Figg WD, et al. Tumor regression and growth rates determined in five intramural NCI prostate cancer trials: the growth rate constant as an indicator of therapeutic efficacy. Clin Cancer Res. 2011; 17:907-17. [PubMed: 21106727]

30. von Mehren M, Arlen P, Tsang KY, Rogatko A, Meropol N, Cooper HS, et al. Pilot study of a dual gene recombinant avipox vaccine containing both carcinoembryonic antigen (CEA) and B7.1 transgenes in patients with recurrent CEA-expressing adenocarcinomas. Clin Cancer Res. 2000; 6:2219-28. [PubMed: 10873071]

31. von Mehren M, Arlen P, Gulley J, Rogatko A, Cooper HS, Meropol NJ, et al. The influence of granulocyte macrophage colony-stimulating factor and prior chemotherapy on the immunological response to a vaccine (ALVAC-CEA B7.1) in patients with metastatic carcinoma. Clin Cancer Res. 2001; 7:1181-91. [PubMed: 11350882]

32. Schlom J, Arlen PM, Gulley JL. Cancer vaccines: moving beyond current paradigms. Clin Cancer Res. 2007; 13:3776-82. [PubMed: 17606707]

33. Hodi FS, O'Day SJ, McDermott DF, Weber RW, Sosman JA, Haanen JB, et al. Improved survival with ipilimumab in patients with metastatic melanoma. N Engl J Med. 2010; 363:711-23. [PubMed: 20525992]

34. Hoos A, Eggermont AM, Janetzki S, Hodi FS, Ibrahim R, Anderson A, et al. Improved endpoints for cancer immunotherapy trials. J Natl Cancer Inst. 2010; 102:1388-97. [PubMed: 20826737]

35. Pelzer U, Schwaner I, Stieler J, Adler M, Seraphin J, Dorken B, et al. Best supportive care (BSC) versus oxaliplatin, folinic acid and 5-fluorouracil (OFF) plus BSC in patients for second-line advanced pancreatic cancer: A phase III-study from the German CONKO-study group. Eur J Cancer. 2011; 47:1676-81. [PubMed: 21565490]

36. Lyerly HK, Hobeika A, Niedzwiecki D, Osada T, Marshall J, Garrett CR, et al. A dendritic cellbased vaccine effects on T-cell responses compared with a viral vector vaccine when administered to patients following resection of colorectal metastases in a randomized phase II study. J Clin Oncol. 2011; 29 suppl; abstr 2533.

37. Morse M, Niedzwiecki D, Marshall J, Garrett CR, Chang DZ, Aklilu M, et al. Survival rates among patients vaccinated following resection of colorectal cancer metastases in a phase II randomized study compared with contemporary controls. J Clin Oncol. 2011; 29 suppl; abstr 3557.

38. Choti MA, Sitzmann JV, Tiburi MF, Sumetchotimetha W, Rangsin R, Schulick RD, et al. Trends in long-term survival following liver resection for hepatic colorectal metastases. Ann Surg. 2002; 235:759-66. [PubMed: 12035031]

39. Pawlik TM, Scoggins CR, Zorzi D, Abdalla EK, Andres A, Eng C, et al. Effect of surgical margin status on survival and site of recurrence after hepatic resection for colorectal metastases. Ann Surg. 2005; 241:715-22. discussion 22-4. [PubMed: 15849507]

40. Sasaki A, Iwashita Y, Shibata K, Matsumoto T, Ohta M, Kitano S. Analysis of preoperative prognostic factors for long-term survival after hepatic resection of liver metastasis of colorectal carcinoma. J Gastrointest Surg. 2005; 9:374-80. [PubMed: 15749600]

41. Andres A, Majno PE, Morel P, Rubbia-Brandt L, Giostra E, Gervaz P, et al. Improved long-term outcome of surgery for advanced colorectal liver metastases: reasons and implications for management on the basis of a severity score. Ann Surg Oncol. 2008; 15:134-43. [PubMed: 17909911]

42. Arru M, Aldrighetti L, Castoldi R, Di Palo S, Orsenigo E, Stella M, et al. Analysis of prognostic factors influencing long-term survival after hepatic resection for metastatic colorectal cancer. World J Surg. 2008; 32:93-103. [PubMed: 18027020] 
43. House MG, Ito H, Gonen M, Fong Y, Allen PJ, DeMatteo RP, et al. Survival after hepatic resection for metastatic colorectal cancer: trends in outcomes for 1,600 patients during two decades at a single institution. J Am Coll Surg. 2010; 210:744-52. 52-5. [PubMed: 20421043]

44. Boehm AL, Higgins J, Franzusoff A, Schlom J, Hodge JW. Concurrent vaccination with two distinct vaccine platforms targeting the same antigen generates phenotypically and functionally distinct T-cell populations. Cancer Immunol Immunother. 2010; 59:397-408. [PubMed: 19756595]

45. Bristol JA, Schlom J, Abrams SI. Persistence, immune specificity, and functional ability of murine mutant ras epitope-specific CD4(+) and CD8(+) T lymphocytes following in vivo adoptive transfer. Cell Immunol. 1999; 194:78-89. [PubMed: 10357883]

46. Gelbard A, Garnett CT, Abrams SI, Patel V, Gutkind JS, Palena C, et al. Combination chemotherapy and radiation of human squamous cell carcinoma of the head and neck augments CTL-mediated lysis. Clin Cancer Res. 2006; 12:1897-905. [PubMed: 16551875]

47. Reits EA, Hodge JW, Herberts CA, Groothuis TA, Chakraborty M, Wansley EK, et al. Radiation modulates the peptide repertoire, enhances MHC class I expression, and induces successful antitumor immunotherapy. J Exp Med. 2006; 203:1259-71. [PubMed: 16636135]

48. Chakraborty M, Wansley EK, Carrasquillo JA, Yu S, Paik CH, Camphausen K, et al. The use of chelated radionuclide (samarium-153-ethylenediaminetetramethylenephosphonate) to modulate phenotype of tumor cells and enhance T cell-mediated killing. Clin Cancer Res. 2008; 14:4241-9. [PubMed: 18594006]

49. Zitvogel L, Kepp O, Kroemer G. Immune parameters affecting the efficacy of chemotherapeutic regimens. Nat Rev Clin Oncol. 2011; 8:151-60. [PubMed: 21364688]

50. Madan RA, Mohebtash M, Arlen PM, Vergati M, Rauckhorst M, Steinberg SM, et al. Ipilimumab and a poxviral vaccine targeting prostate-specific antigen in metastatic castration-resistant prostate cancer: a phase 1 dose-escalation trial. Lancet Oncol. 2012; 13:501-8. [PubMed: 22326924]

51. Bilusic M, Gulley J, Heery C, et al. A randomized phase II study of flutamide with or without PSA-TRICOM in nonmetastatic castration-resistant prostate cancer. J Clin Oncol. 2011; 29 suppl 7; abstr 163.

52. Anderson P, Nunez R. Samarium lexidronam (153Sm-EDTMP): skeletal radiation for osteoblastic bone metastases and osteosarcoma. Expert Rev Anticancer Ther. 2007; 7:1517-27. [PubMed: 18020921]

53. Heery, CR.; Madan, RA.; Bilusic, M., et al. Interim analysis of a phase 2 randomized clinical trial of samarium-153 (Sm-153) with or without PSA-TRICOM vaccine in metastatic castrationresistant prostate cancer after docetaxel. Abstract accepted for publication. ASCO Annual Meeting; Chicago, IL. June 1-5, 2012; [JCO 30(15), May 20 suppl, 2012, abstr 2526]

54. [Accessed April 11,2011] Docetaxel alone or in combination with vaccine to treat breast cancer. http://www.clinicaltrials.gov/ct2/show/NCT00179309?term=gulley\&rank=9

55. Petrylak, D. Defining the optimal role of immunotherapy and chemotherapy: Advanced prostate cancer patients who receive sipuleucel-T (PROVENGE) followed by docetaxel derive greatest survival benefit. 14th Annual Meeting of the Chemotherapy Foundation Symposium; New York, NY. November 8-11; 2006.

56. Gribben JG, Ryan DP, Boyajian R, Urban RG, Hedley ML, Beach K, et al. Unexpected association between induction of immunity to the universal tumor antigen CYP1B1 and response to next therapy. Clin Cancer Res. 2005; 11:4430-6. [PubMed: 15958627]

57. Antonia SJ, Mirza N, Fricke I, Chiappori A, Thompson P, Williams N, et al. Combination of p53 cancer vaccine with chemotherapy in patients with extensive stage small cell lung cancer. Clin Cancer Res. 2006; 12:878-87. [PubMed: 16467102]

58. Arlen PM, Gulley JL, Parker C, Skarupa L, Pazdur M, Panicali D, et al. A randomized phase II study of concurrent docetaxel plus vaccine versus vaccine alone in metastatic androgenindependent prostate cancer. Clin Cancer Res. 2006; 12:1260-9. [PubMed: 16489082]

59. [Accessed March 29, 2011] Docetaxel and prednisone with or without vaccine therapy in treating patients with metastatic hormone-resistant prostate cancer. http://www.clinicaltrials.gov/ct2/show/NCT01145508?term=McNeel\&rank=5 
60. Jacoby RF, Seibert K, Cole CE, Kelloff G, Lubet RA. The cyclooxygenase-2 inhibitor celecoxib is a potent preventive and therapeutic agent in the min mouse model of adenomatous polyposis. Cancer Res. 2000; 60:5040-4. [PubMed: 11016626]

61. Steinbach G, Lynch PM, Phillips RK, Wallace MH, Hawk E, Gordon GB, et al. The effect of celecoxib, a cyclooxygenase-2 inhibitor, in familial adenomatous polyposis. N Engl J Med. 2000; 342:1946-52. [PubMed: 10874062]

62. Zeytin HE, Patel AC, Rogers CJ, Canter D, Hursting SD, Schlom J, et al. Combination of a poxvirus-based vaccine with a cyclooxygenase-2 inhibitor (celecoxib) elicits antitumor immunity and long-term survival in CEA.Tg/MIN mice. Cancer Res. 2004; 64:3668-78. [PubMed: 15150127]

63. Dahut WL, Gulley JL, Arlen PM, et al. Randomized phase II trial of docetaxel plus thalidomide in androgen-independent prostate cancer. J Clin Oncol. 2004; 22:2532-2539. [PubMed: 15226321]

64. Tannock IF, de Wit R, Berry WR, et al. Docetaxel plus prednisone or mitoxantrone plus prednisone for advanced prostate cancer. N Engl J Med. 2004; 351:1502-12. [PubMed: 15470213]

65. de Bono JS, Oudard S, Ozguroglu M, et al. Prednisone plus cabazitaxel or mitoxantrone for metastatic castration-resistant prostate cancer progressing after docetaxel treatment: a randomised open-label trial. Lancet. 2010; 376:1147-54. [PubMed: 20888992]

66. de Bono JS, Logothetis CJ, Molina A, et al. Abiraterone and increased survival in metastatic prostate cancer. N Engl J Med. 2011; 364:1995-200. [PubMed: 21612468]

67. Gulley JL, Madan RA, Tsang KY, et al. A pilot safety trial investigating a vector-based vaccine targeting carcinoembryonic antigen in combination with radiotherapy in patients with gastrointestinal malignancies metastatic to the liver. Expert Opin Biol Ther. 2011; 11:1409-18. [PubMed: 21871012] 

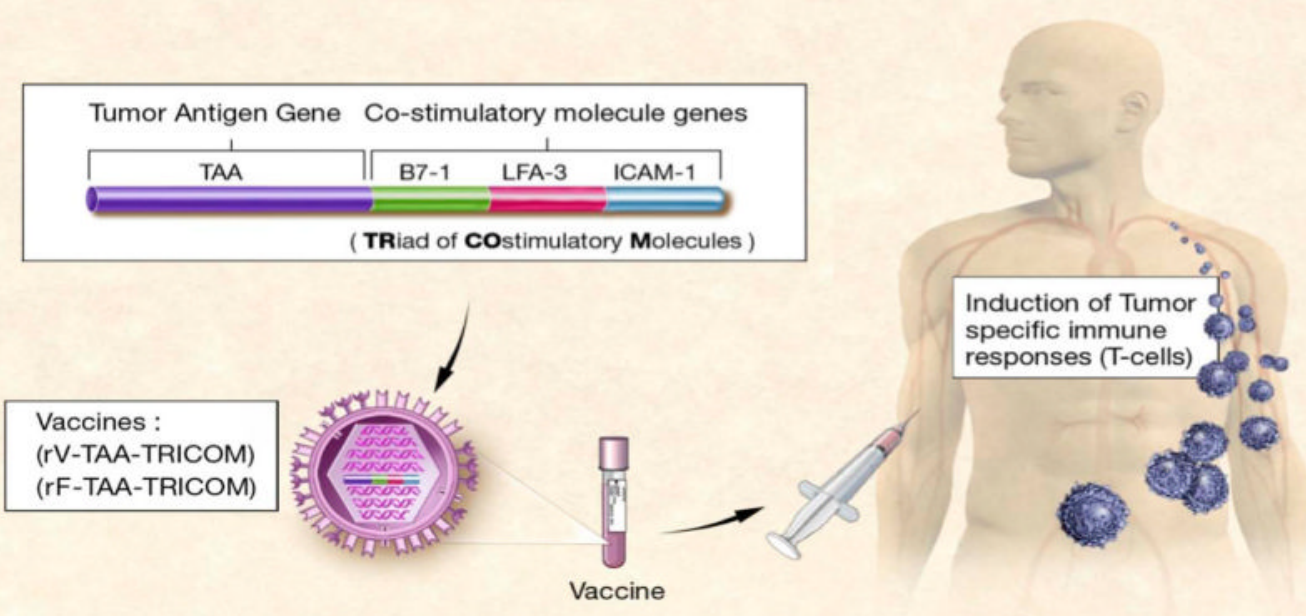

Figure 1. TRICOM Therapeutic Cancer Vaccine Platform

The TRICOM vaccination platform consists of a single recombinant vaccinia $(\mathrm{rV}-)$ prime vaccination followed by six or more monthly booster vaccinations with recombinant fowlpox (rF-). Each of these vectors contains transgenes for one or more tumor-associated antigens and transgenes for three human T-cell costimulatory molecules (designated TRICOM) to enhance T-cell activation to the tumor antigens. ${ }^{6-7,15}$ For prostate cancer trials, rV-, rF-PSA-TRICOM (also designated PROSTVAC) is employed ${ }^{53,3,20}$ For trials of other carcinomas (breast, colorectal, etc.), the pan-carcinoma antigen transgenes for carcinoembryonic antigen CEA and MUC-1 are used along with TRICOM, i.e., rV-, rFCEA-MUC-1-TRICOM (designated PANVAC). ${ }^{54,59,17}$ Vaccinations are given subcutaneously. 


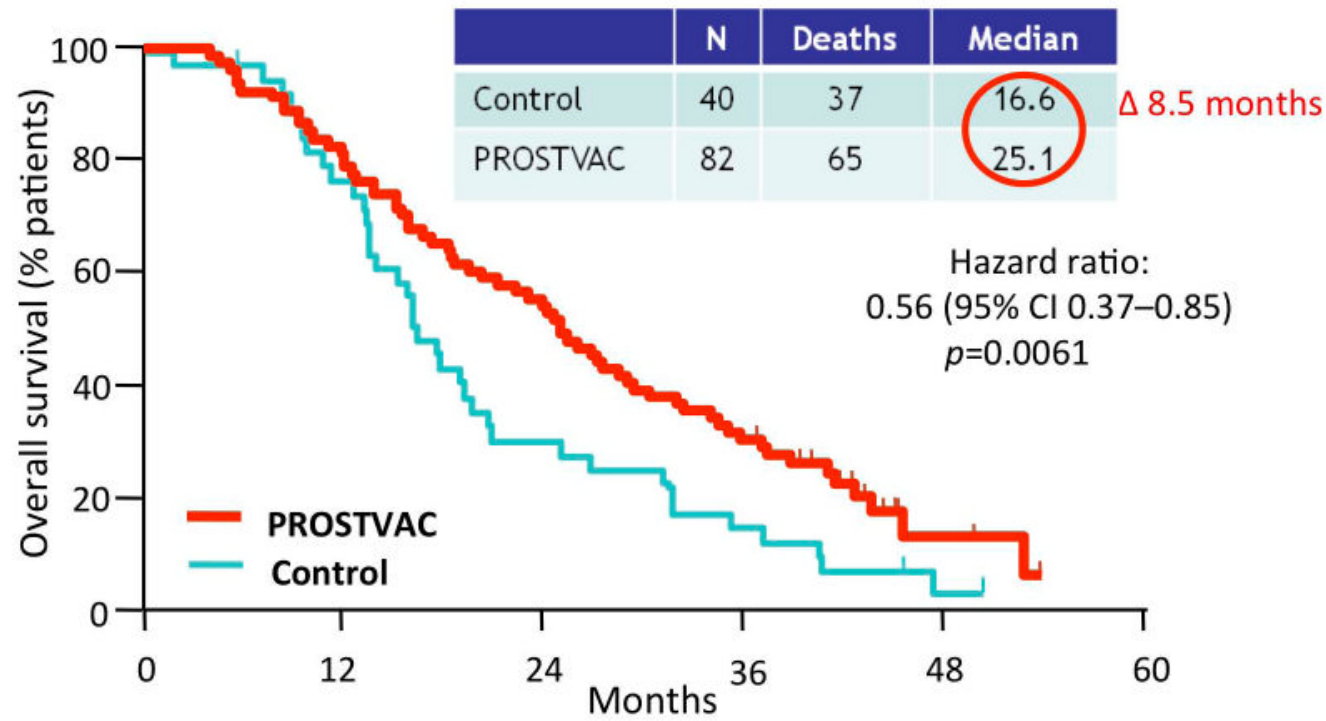

Figure 2. Overall Survival Advantage Using Cancer Vaccines

A, Overall survival of a randomized, placebo (empty vector) controlled 43-center trial of PSA-TRICOM vaccine in patients with metastatic castrate-resistant prostate cancer. ${ }^{20}$ There was an overall survival advantage of 8.5 months $(p=0.006)$ and a $44 \%$ reduction in death in the vaccine arm. Adapted from Kantoff et al. ${ }^{20}$ 

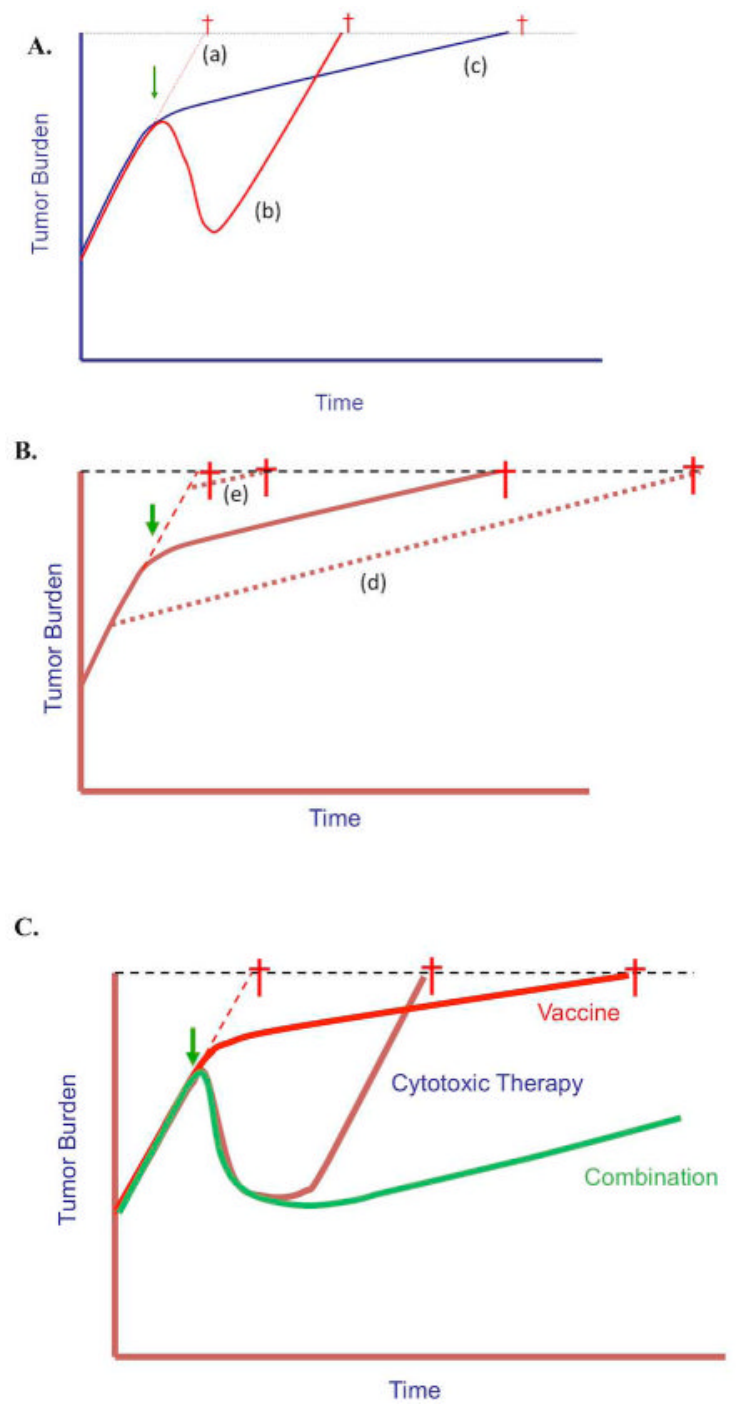

Figure 3. Tumor Growth Rates Following Chemotherapy vs. Vaccine Therapy

Adapted from data in ref. 29. A, Growth rate of tumor if no therapy is initiated (a). An examination of five clinical trials (four with chemotherapy and one with PSA-TRICOM vaccine) in patients with mCRPC demonstrated that with the use of chemotherapy there was an initial tumor reduction, but that the growth rate of tumors at relapse (b) was similar to the initial tumor growth rate prior to therapy; this is contrasted with the reduction in tumor growth rate following vaccine therapy (c). Thus for patients with little or any tumor reduction (and thus virtually no increase in time to progression), an increase in survival was observed. B, This phenomenon could potentially be enhanced if vaccine therapy is initiated earlier in disease progression or in patients with low tumor burden metastatic disease (d), but would have minimal effect in patients with large tumor burden (e). C, Additional therapies received with vaccine may take advantage of both modalities. $\dagger$, projected time of death. 
Table 1

Predicted Survival by Halabi Nomogram Score vs. Actual Survival

\begin{tabular}{lccc}
\hline & All patients & $\begin{array}{c}\text { Patients with Halabi } \\
\text { predicted survival < 18 } \\
\text { mos }\end{array}$ & $\begin{array}{c}\text { Patients with Halabi } \\
\text { predicted survival } \geq \mathbf{1 8 ~ m o s}\end{array}$ \\
\hline $\begin{array}{l}\text { Vaccine: PROSTVAC (n=32) } \\
\text { Predicted survival by Halabi score (mos) }\end{array}$ & 17.4 & 12.3 & 20.9 \\
\hline Actual median overall survival (mos) & 26.6 & 14.6 & $\begin{array}{c}\text { Not reached (8 of 15 pts alive } \\
\text { at 37.3 mos) }\end{array}$ \\
\hline $\begin{array}{l}\text { Difference (mos) } \\
\text { Patients' survival longer than predicted by Halabi }\end{array}$ & 9.2 & 2.3 & $\mathbf{1 2}$ of 15 (80\%) P=0.035 \\
\hline $\begin{array}{l}\text { Domogram } \\
\text { Docetaxel therapy (n=22) }\end{array}$ & 22 of 32(69\%) & 10 of $17(59 \%)$ & 21.0 \\
\hline Predicted survival by Halabi score (mos) & 16.5 & 13.0 & 16.9 \\
\hline Actual median overall survival (mos) & 15.5 & 15.4 & $(-4.1)$ \\
\hline $\begin{array}{l}\text { Difference (mos) } \\
\text { Patients' survival longer than predicted by Halabi }\end{array}$ & $(-1.0)$ & 2.4 & 3 of $9(33 \%)$ \\
\hline
\end{tabular}

Results of two contemporary phase II trials in metastatic castration-resistant prostate cancer (mCRPC) at the NCI. The median overall survival in the vaccine trial was 26.6 months, similar to the larger randomized PROSTVAC (PSA-TRICOM) trial. A retrospective analysis indicated that patients with more indolent disease characteristics and a predicted survival greater than 18 months using the Halabi mCRPC nomogram, ${ }^{27}$ had greater improvements in survival (compared to predicted on chemotherapy or second-line hormonal therapy) than patients with more aggressive disease characteristics. This is contrasted with the contemporary trial at NCI with a similar patient population in which the Halabi nomogram more accurately predicted response to docetaxel chemotherapy. 63 


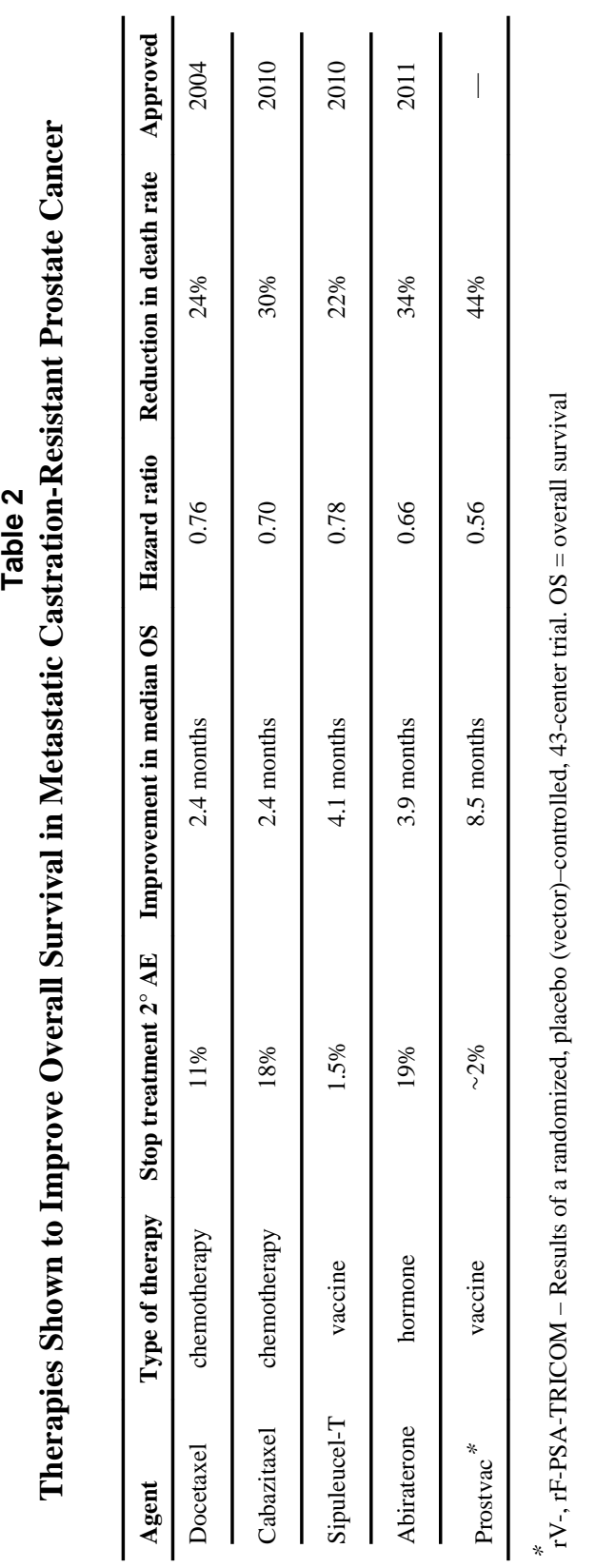

